\title{
Tensile Test of GFRP Bar Using Machine Vision
}

Zujia Zheng ${ }^{1,2}$, Xue $\mathrm{Yang}^{1}$, Zhigang Chen ${ }^{1}$ and Mingchao $\mathrm{Yu}^{2}{ }^{2} \mathrm{a}^{*}$

${ }^{1}$ Infrastructure and Logistics Management, Wuhan University of Science and Technology, Wuhan 430065, China

${ }^{2}$ Key Laboratory of Metallurgical Equipment and Control Technology, Ministry of Education, Wuhan University of Science and Technology, Wuhan 430081, China.

a*503616860@qq.com

\section{Keywords: GFRP bar; Machine vision; Tensile test; Strain measurement}

\begin{abstract}
With the development of modern industrial technology, the efficiency and accuracy of material performance measurement are required to be higher and higher. The performance of materials plays a decisive role in the quality of the engineering, so it is becoming more and more important to test the properties of the materials. Because of the difference between the GFRP bar and the general reinforcement material, the mechanism of the distribution and development of cohesive force between GFRP bar and grouting material is also a problem which is urgent for scientific research workers to solve. In order to overcome the shortcomings of traditional extensometer strain measurement method in metal material tensile test, a non-contact strain precise measurement is carried out based on machine vision measurement principle. At present, many scholars have studied the contactless measurement based on machine vision and achieved some results. However, there are still many problems to be studied and improved in practical industrial applications. According to the performance requirement of metal materials and the principle of machine vision measurement, the non-contact strain measurement method has been further studied, and a complete strain measurement system has been developed.
\end{abstract}

\section{Introduction}

With the development of industrial technology, the performance requirements of materials are also higher and higher. In order to get the real performance parameters, more and more requirements are put forward for the detection methods of materials. For most materials, the essential testing index is stress and strain [1]. The extensometer is the basic device for measuring the axial and radial deformation of the specimen, which is often used in the measurement of material strain. For the measurement of tensile or compression deformation of metal materials, it is commonly used as a contact extensometer. It is easy to produce friction with the specimen when the material is stretched or compressed, which causes the measurement error, especially the rigid material [2]. When the specimen breaks, it will cause severe vibration and impact, which will seriously damage the extensometer. Therefore, the traditional contact extensometer cannot measure the whole process of material tensile deformation. The extensometer must be unloaded before the specimen breaks, so as not to damage the extensometer. The glass fiber composite material is a glass fiber composite material with different properties, based on the polymer matrix and different properties and different components of glass fiber materials. The GFRP bar has the advantages of light weight, high strength and good corrosion resistance. If we can use GFRP soil nail in supporting engineering, we can not only save the amount of steel and reduce the cost, but also give full play to the excellent performance of glass fiber composite [3].

\section{The hardware system of machine vision}

The design of the machine vision strain measurement system mainly includes two parts, that is, the hardware component of the system and the component part of the software. The hardware part is mainly industrial CCD camera, which provides illumination environment for image sensor and 
lighting system, and PC machine provides hardware basis and test machine for image processing [4] The hardware system is the foundation of rapid measuring system can accurately measure, it can decide whether the system can collect sufficiently clear image and image transmission speed, for subsequent image processing to obtain enough measurement points and painting displacement strain curves provide accurate assurance. The rapidity and accuracy of strain measurement system is determined by the speed of computer operation, the speed of image algorithm processing, the speed of data transmission and the size of image information. The deformation measurement system is composed of lighting system, image acquisition module, image processing module, data processing and display module [5], as shown in Figure 1.

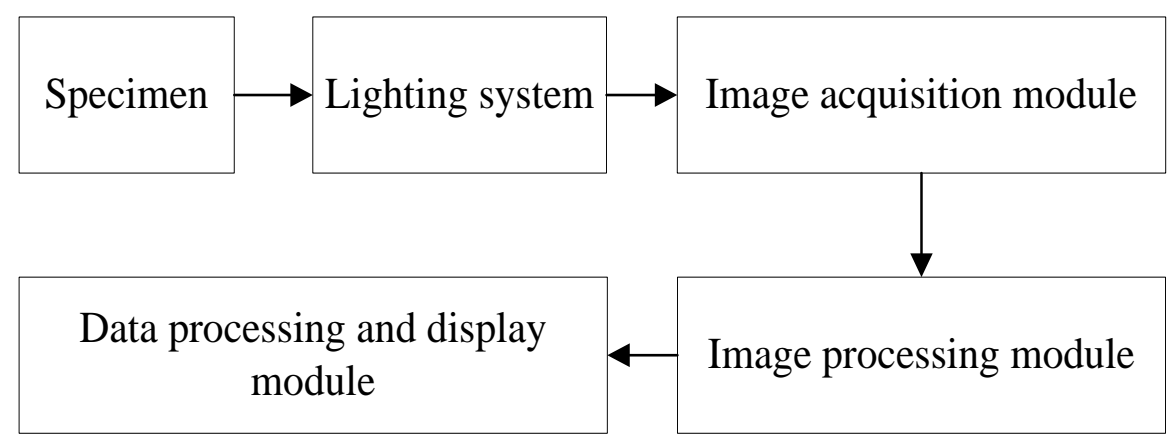

Figure 1. System composition block diagram

After the analysis of the function of the system, two aspects of the design of hardware and software should be considered for the design of the system overall plan. The hardware includes the lighting system, the CCD camera, the test machine, the test piece and the PC machine. The design of these hardware parts will greatly influence the measurement accuracy and measurement speed of the measurement system. In order to improve the measurement accuracy and speed of the system, in addition to improving the configuration of hardware devices, we can also improve the effectiveness of the system through the design of software part [6].

\section{Mechanical properties of GFRP tendons}

The axial tension along the direction of the nail body is the main bearing of the soil nailing in the support process. Therefore, the premise of using GFRP bars instead of reinforcing bars in soil nailing is to have enough strength in the tensile process of GFRP bars, and the mechanical properties and failure modes of GFRP bars will also affect the normal application of GFRP soil nailing [7]. The basis of the normal use of GFRP soil nailing is that the reinforcement and mortar can bear the load together, and the bond force between the reinforcement and the mortar is the precondition for the soil nailing to bear the load. Only the bond strength between the GFRP bar and the mortar can ensure the joint stress and deformation of the GFRP reinforcement and the mortar [8].

The damage of glass fibers in the GFRP tendons is often caused by the weak "weak" fibers with defects. When some fibers in the reinforcement body break and form cracks, there will be local stress disturbance near the part of the reinforcement, resulting in concentrated stress. A simplified model is established by using the theory of shear lag to analyze and solve the phenomenon of stress redistribution. GFRP bar and mortar body are two kinds of materials with completely different characteristics [9]. If we need to combine GFRP bars with mortar, we must ensure enough cohesion between GFRP bar and mortar body. Only if there are reliable bonding and anchoring between them, can we ensure the stress transfer at the interface between GFRP bar and mortar body, and ultimately meet the working stress of the whole structure.

In 1961, Hwdgepeth first used shear lag theory to deal with the fiber fracture problem of unidirectional composites, and the plastic effect of the matrix and the problem of interfacial 
degumming are studied. But the premise of using this method is to put forward the following assumptions: 1) All the displacement is parallel to the direction of the fiber; 2) In the whole process of stress, the fiber is only pulled by tension; 3) In the whole process, the matrix can only transfer the shear force and do not bear the tension [9].

\section{Calibration of measurement system}

For the machine vision based measurement system, calibration is very necessary and very important. If the system is not calibrated or inaccurate, it is meaningless to measure the data measured by the system. For the design measurement system, the accuracy of measurement is mainly determined by the accuracy of the results of the software processing and the calibration of the system. The accurate measurement of the measurement system is the prerequisite for accurate measurement [11].

According to monocular vision imaging principle, as shown in Figure 2, when the object A is moving in the same plane or in the same plane, the object and its image a meet the similar relationship. As long as the length of $\mathrm{L} 1$ extracted from the image L1, multiplied by a magnification $\mathrm{k}$ to get the actual length of the object A L2. And the magnification $\mathrm{K}$ can be obtained by calibration. So monocular vision measurement needs only one CCD camera to achieve measurement [12]. It is simple and convenient, and has unique advantages in two-dimensional measurement.

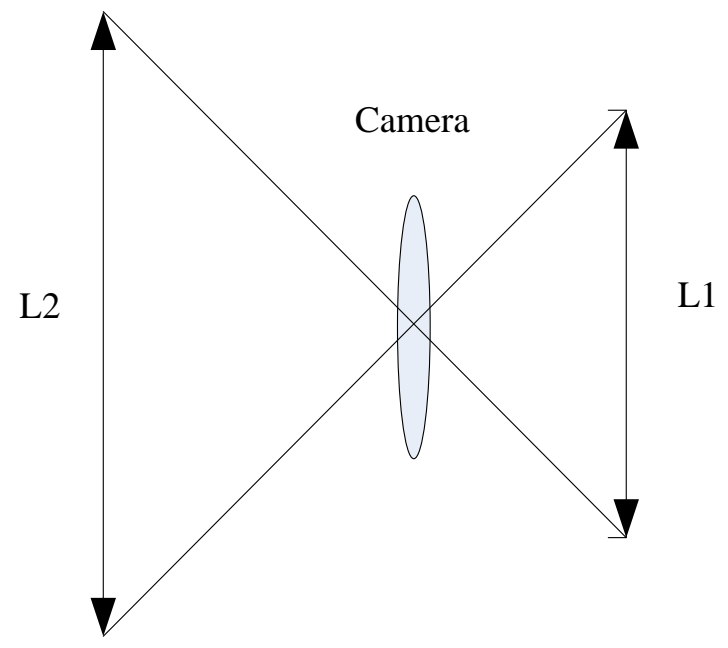

Figure 2. Principle of monocular imaging

\section{Image recognition and processing}

The edge detection by edge detection is a set of discrete points, which are not in the same line, but constitute an irregular curve. For the strain measurement system, it is difficult to find the distance between the two irregular curves [13]. These curves need to be drawn into lines, and the distance between the lines is calculated. The commonly used line detection methods are least squares linear fitting, Hough transformation and so on.

In the use of digital image acquisition system to obtain images, there will be different degrees of external interference and internal interference, and these interference cannot be avoided. And in the process of image acquisition, there will be a certain degree of pixel jitter, which will reduce the quality of the image. However, the degradation of image quality will be unfavorable to the high accuracy measurement of edges. Because the grayscale of this image is no longer as uniform and continuous as it used to be, but it will suddenly become larger or smaller, making the image form some false edges and contours, so that the measurement is not accurate [14]. Therefore, in order to restore the original image, it is necessary to remove the noise of the image. This study uses the image filtering method to remove the noise of the image. The common filtering methods include 
neighborhood mean filter, Gauss filter and median filter. Each of these filtering methods is introduced, and the three filtering methods are used to denoise the images respectively, and their respective filtering effects are compared.

Neighborhood mean filtering. The neighborhood mean filtering is also called the spatial domain average filtering. The mean filtering process of the image is as follows: A rectangle window $\mathrm{S}$ is sliding on the image, and the average value of the gray value of each pixel point in the window's center pixel corresponds to the gray value of the pixel, and the average gray value of the pixel is replaced by the average value. One thing to note is that, in the process of scanning images with windows, the four edges of pixels cannot be processed, or the edges of the image can be extended by using pixels with a gray value of 0 [15].

Gauss filter. Gauss smoothing filter is also the idea of using neighborhood mean filtering, which is different from neighborhood mean filtering. Gauss filter performs weighted averaging on image neighborhood. Is generally believed that the influence of the pixel and window center pixel template closer to the filtering result is bigger, so the coefficient of pixels from the center near the template should be larger, according to the template in each pixel size center pixel distance is different, with different weight average. In this way, when the template is filtered, the image details can be blurred and more image gray distribution features can be retained [16].

Median filtering. Median filter is a nonlinear signal processing method. The basic principle is that the gray value of each pixel in the image is replaced by the median of the gray value of all the pixels in the neighborhood of the point.

\section{Conclusions}

In the field of construction engineering, material change is crucial to the advancement and development of the construction industry. Soil nailing is a common supporting method in slope engineering and foundation pit engineering, which has been widely used in the world. The most common soil nailing in the project is still the steel soil nailing which is formed by grouting in rock and soil. Due to the corrosion resistance and poor durability of the steel, a large amount of loss has been brought to the actual project. Glass fiber composite material has the characteristics of light quality, high strength, and good corrosion resistance and so on. The use of GFRP bar instead of steel bar as the reinforcement material of soil nailing can not only save steel bars, reduce costs, but also give full play to the special properties of composite materials. So it is very important to study the mechanics of GFRP bars. The real-time measurement system based on machine vision has the advantages of simple operation, high accuracy and little environmental impact. It is believed that it will be widely studied and applied in the future.

\section{References}

[1] Q.P. Jin, Z.J. Zheng and L.L. Xiao. Engineering Plastics Application, (2014) No.7, p.82

[2] Q.J. Jin, Z.J. Zheng, W. Lu and Z. Chen. China Plastics, Vol. 28 (2014) No.11, p.67.

[3] Z.J. Zheng. Wuhan University Of Science And Technology, (2015)

[4] W. Tian and Z.J. Zheng. The World of Building Materials, (2015) No.1, p.25-28.

[5] Q.P. Jin, Z.J. Zheng and X.W. Lei. Construction Technology, (2014) No.19, p.94-97.

[6] Q.P. Jin, Z.J. Zheng, Z. Chen and X.Q. Li. Plastics industry, Vol. 42 (2014) No.10, p.50-54.

[7] J. Gao. Harbin Institute Of Technology, (2006).

[8] H.F. Pei, J.H. Yin, H.H. Zhu, C.Y. Hong and Y.H. Fan. Journal of rock mechanics and Engineering, Vol. 29 (2010) No.8, p.1570-1576.

[9] Q. Zhang, H.L. Pei, B.H. Shi and Y.J. Zhao. Journal of South China University of Technology (Social Science Edition), Vol. 40 (2012) No.3, p.81-87.

[10]P. Zhao, X.F. Zhao, Q.A. Zhao, S.Y. Li, T.W. Fang and X.R. Zhao. Internet of Things, Vol. 7 (2017) No.9, p.14-18.

[11] J.G. Sun, N. Gao, D. Yang and T.J. Li. Journal of Hebei University of Technology, Vol. 42 (2013) No.4, p.64-68. 
[12]H.F. Li and H.C. Wang. Computer application, Vol. 34 (2014) No.9, p.2557-2561.

[13]Z. Tian, Q. Zhang, J.L. Xiong and G.C. Wang. Acta Optica Sinica, Vol. 31 (2011) No.4, p.186-191.

[14] Y.H. Sun, C.B. Yi, H. Pi and S.Y. Guo. Polymer notification, (2011) No.12, p.128-132.

[15]Z.B. Zhu. Railway Investigation and Surveying, Vol. 38 (2012) No.1, p.88-91.

[16] Q.D. Hao, C. Wang, B. Wang and J.P. Ou. Journal of Harbin Institute of Technology, Vol. 44 (2012) No.2, p.7-10. 